\title{
The Utility of Somatosensory Evoked Potential Monitoring During Cervical Spine Surgery: How Often Does It Prompt Intervention and Affect Outcome?
}

\author{
Michael S. Roh*, Tracy J. Wilson-Holden ${ }^{\dagger}$, Anne M. Padberg $^{\dagger}$, \\ Jong-Beom Park ${ }^{\dagger}$, K. Daniel Riew ${ }^{\ddagger}$ \\ ${ }^{*}$ Rockford Orthopedic Associates, Rockford, Illinois, ${ }^{\dagger}$ BJC Health Systems, St. Louis, Missouri, \\ ${ }^{\ddagger}$ Washington University School of Medicine
}

\begin{abstract}
Study Design: Retrospective review of the results of somatosensory evoked potentials (SSEP) performed in cervical spine surgery.

Purpose: To evaluate the utility of spinal cord monitoring during cervical spine surgery in a single surgeon's practice, based on how often it prompted an intraoperative intervention.

Overview of Literature: Intraoperative monitoring during cervical spine surgery is not a universally accepted standard of care. This is due in part to the paucity of literature regarding the impact of monitoring on patient management or outcome. Methods: SSEP for tibial, median, and ulnar nerves were monitored in 809 consecutive cervical spine operations performed by a single surgeon. The average patient age was 52 years (range, 2 to 88 years), with 472 males and 339 females. Cases were screened for significant degradation or loss of SSEP data. Specific attention was paid to 1) what interventions were performed in response to the SSEP degradation with subsequent improvement, and 2) whether SSEP changes corresponded with postoperative neurological deficits.

Results: Seventeen of 809 patients (2.1\%) had SSEP degradation that met warning criteria and therefore prompted intervention. Release of shoulder tape (8) or traction (4) most often resulted in SSEP improvement. Failure of SSEP data to return to within acceptable limits of baseline was associated with neurological deficit $(p=0.04)$. Two patients awoke with new postoperative neurological deficits, which resolved in 6 hours and 2 months respectively. Patients with ossification of the posterior longitudinal ligament (OPLL) were at seven-fold greater risk of intraoperative SSEP degradation.

Conclusions: SSEP monitoring in this surgical population proved sensitive to perioperative factors which may increase the risk of postoperative neurologic deficit, and probably prevented neurological deficits in 15 of 809 patients (1.9\%). Improvement in data following intervention appears to correlate well with unchanged neurologic status. Experience with intraoperative monitoring in this patient series has led to incorporation of these techniques as a standard of care in cervical spine surgeries performed by this surgeon.
\end{abstract}

Key Words: Somatosensory evoked potentials, Cervical spine surgery, Postoperative neurological deficits

\section{Introduction}

Intraoperative neurophysiologic monitoring during cervical spine surgery is not a universally accepted standard of care. Proponents of its usage have reported a lower inci- dence of quadriplegia ${ }^{1}$ and potentially helpful prognostic information ${ }^{2.3}$. Some surgeons believe that spinal cord monitoring may decrease the incidence of postoperative brachial plexopathy following cervical spine surgery, though to date, this has only been shown in thoracic and lumbar deformity surgery ${ }^{4,5}$. Despite favorable reports in the literature, many

Corresponding author: K. Daniel Riew, MD

Suite 11300 One Barnes-Jewish Hospital Plaza, St. Louis, MO 63110

Tel: +1-314-747-2565, Fax: +1-314-747-2599, E-mail: riewd@wustl.edu 
surgeons question whether the added time and expense involved in monitoring is justified. These issues are particularly salient when considering the use of monitoring for even routine cervical spine cases.

The utility of a surgical adjunct such as a spinal cord monitoring may be evaluated by how its use leads to an intraoperative intervention that maintains or improves postoperative neurological function. Several studies have investigated the use of somatosensory-evoked ${ }^{1-3,6,7}$ and motorevoked $^{8-10}$ potential monitoring for cervical spine cases. However, few published studies have focused on how monitoring impacts intraoperative management or patient care in cervical spine surgery ${ }^{1,2}$.

For the past five years, it has been the policy of the senior author to use intraoperative monitoring for all cervical spine operations. The purpose of this paper was to review a large series of cervical spine operations by a single surgeon to evaluate the frequency and manner in which spinal cord monitoring prompted an intraoperative intervention that affected outcome. To our knowledge, this represents the largest published series of cervical spine cases with SSEP monitoring data.

\section{Materials and Methods}

\section{Clinical data}

Eight hundred and nine consecutive cervical spine operations were performed with the use of intraoperative somatosensory evoked potentials (SSEP) monitoring. All of these cases were performed by a single surgeon. The cases were described as follows: anterior (398), posterior (244), anterior-posterior (82), and anterior-posterior-anterior (1).

Cervical procedures were performed for cervical spondylotic radiculopathy and myelopathy, cervical disc disease, rheumatoid arthritis, ossification of the posterior longitudinal ligament (OPLL), trauma, and tumor. The average patient age was fifty-two years (range, 2 to 88 years), with 472 males and 339 females.

Patients were positioned supine on a closed-frame Jackson table for anterior procedures, and prone on an openframe Jackson table (OSI Medical, Union City, CA, USA) for posterior procedures. For all cases, the surgical team applied 3" silk tape from the patients' shoulders to the foot of the Jackson table with only enough tension to gently depress the shoulders. The arms were strapped to the patients' sides with linen sheets that were either tucked beneath the patient (anterior cases) or taped together across the patient (posterior cases). Care was taken to pad all bony prominences. Ten to twenty pounds of traction were applied via Gardner-Wells tongs for all posterior cases, and on anterior cases until 1999. Patients were placed in a reverse Trendelenburg position for posterior cases. Based on the senior author's personal preference, intraoperative hypotension was not used.

Cases were carefully screened for the presence of intraoperative deterioration of SSEP data. Specific attention was paid to 1) what interventions were performed in response to the deterioration of SSEP data, and 2) whether the decline in SSEP data corresponded with a new postoperative neurological deficit. Cases were also classified, based on these data. True positive cases had intraoperative SSEP data degradation that correlated with a new postoperative deficit, whereas presumed positive cases had SSEP degradation that improved with interventions and had no new postoperative deficit. False positive cases had intraoperative SSEP degradation that did not improve with interventions, but had no new postoperative deficit. Hospital and office charts, as well as electrophysiology monitoring logs were reviewed to obtain preoperative, postoperative, and follow-up clinical, neurologic, and electrophysiologic data.

\section{Spinal cord monitoring technique}

Intraoperative SSEP was performed on all patients included in the study using the Nicolet Viking series equipment (Nicolet Biomedical, Madison, WI, USA). Lower extremity SSEPs were obtained with stimulation of the posterior tibial nerve at the medial malleolus. The ulnar nerve at the cubital tunnel and the median nerve at the wrist were used for upper extremity monitoring. Pre-gelled patch electrodes (Nicolet Biomedical) were used for stimulation at the aforementioned sites. Stimulation consisted of constant current at an intensity of $30 \sim 35 \mathrm{~mA}$, a rate of $4.7 \sim 5.1 \mathrm{~Hz}$ and duration of $0.3 \mathrm{msec}$.

Far-field responses were recorded from the somatosensory cortex at C3', Cz' and C4', according to the International Federation 10 20 electrode system 9 . A subcortical recording was obtained from the occiput. All responses were recorded with 0.5 inch subdermal needle electrodes (Nicolet Biomedical). Responses consisted of a minimum of 300 averaged trials. Filter settings were $10 \sim 250 \mathrm{~Hz}$ for cortical sites and 10 2,000 $\mathrm{Hz}$ for the subcortical site. Amplifier 
sensitivity ranged from $20 \sim 100 \mathrm{uV}$.

Data were collected prior to incision, once the patient was positioned on the operative table. Baseline latency and amplitude measures were obtained once exposure was complete. These data were used as the control for each patient. All data for upper and lower extremities were collected at approximately five to ten-minute intervals throughout the surgical procedure. The warning criteria used to indicate a significant change in SSEP data were sixty percent decrease in amplitude and/or ten percent increase in latency from all recording sites. Technical and anesthetic variables were ruled out before a warning was given to the surgeon.

General anesthesia was maintained with one of two techniques. The first technique consisted of nitrous oxide at a concentration of fifty percent or less with oxygen mixture, $0.5 \%$ or less exhaled concentration of isofluorane, and a pre-induction bolus injection of morphine. Vecuronium was given as a bolus injection to achieve muscle relaxation. The second technique consisted of induction with $2 \mathrm{mg} / \mathrm{kg}$ of both propofol and ketamine. Continuous drip infusions of each drug were used at a rate of $50 \mu \mathrm{g} / \mathrm{kg}$ per minute for the first hour, reduced to $25 \mu \mathrm{g} / \mathrm{kg}$ per minute thereafter, until wound closure. D-Tubocurarine was used to maintain muscle relaxation.

The response of degraded SSEP data to intraoperative interventions were characterized relative to baseline data. A good improvement signified a return of SSEP data to within acceptable limits of baseline. A fair improvement represented an improvement in SSEP data, but not to within acceptable limits of baseline. Lastly, a poor improvement indicated essentially no SSEP improvement, despite intraoperative maneuvers.

\section{Statistical analysis}

Statistical analysis was performed to identify predisposing factors that may increase the risk of an intraoperative change in SSEP data. Using logistic regression, odds ratios were calculated for age, type of surgery, and the various diagnoses. Also analyzed was the relationship between the degree of intraoperative SSEP improvement following intervention and the presence of a new postoperative neurological deficit.

\section{Results}

Seventeen cases $(2.1 \%)$ were identified in which SSEP data deteriorated and met warning criteria. After technical and anesthetic variables were ruled out, the operative team was notified. The maneuvers that led to improvement in SSEP data included: tape release (8), traction release (4), patient repositioning (2), surgical decompression (2), and truncation of surgery (1). There were eleven male and six female patients, with an average age of fifty-seven years (range, 42 to 89 years). Though many patients had multiple diagnoses, the spectrum of diagnoses for this group of patients included cervical spondylotic disease with myeloradiculopathy (3), myelopathy (1), or radiculopathy (5), herniated nucleus pulposus (4), pseudarthrosis (2), ossification of the posterior longitudinal ligament (5), osteoarthrosis (2), tumor (1), and fracture (1).

Using logistic regression analysis, patients with OPLL were found to be over seven times more likely to have an intraoperative event (odds ratio $=7.44,95 \% \mathrm{CI}=2.51-22.10$, $\mathrm{p}=0.002$ ), as compared to patients without OPLL. With the numbers available, the presence of myelopathy or myeloradiculopathy did not significantly increase the risk of intraoperative SSEP degradation ( $\mathrm{p}=0.34)$.

Two true positive cases were identified ( 2 of $809,0.2 \%$ ), where a new postoperative deficit accompanied the degradation in intraoperative SSEP data. In 15 presumed positive cases $(15$ of $809,1.9 \%)$, the SSEP data degraded but improved following intraoperative interventions, and no new postoperative deficit was noted. No false positive cases (SSEP degradation, improvement, new neurological deficit) were observed.

SSEP data improved to within acceptable limits of baseline following an intervention in 14 of the 17 true and presumed positive cases. Notably, none of these 14 patients with good SSEP improvement had any new postoperative neurological deficit. Three patients had fair or poor improvement in their SSEP data despite interventions, and 2 of these 3 had new postoperative deficits. The first patient had poor improvement following tape release, but awoke with a complete brachial plexopathy. After twenty-four hours, an ulnar neuropathy persisted, which resolved by two months postoperatively. The second patient had fair improvement following tape release, and presented with right-sided hemiparesis in the post-anesthesia care unit, which resolved within six hours. The third patient had degradation of cortical data, for which the operation was abbreviated and a halo vest was placed. Though this patient had no postoperative neurological deficit, it was determined that a transient ischemic attack was the likely cause for 
degradation of monitoring data.

Statistical analysis was performed to analyze the relationship between the degree of intraoperative SSEP improvement following intervention and the presence of a new postoperative neurological deficit. Based on logistic regression results, patients with fair or poor intraoperative SSEP improvement following an intervention are at significantly greater risk of having a postoperative deficit as compared to patients with good SSEP improvement, i.e., back to baseline $(\mathrm{p}=0.04$, odds ratio $=26.00,95 \% \mathrm{CI}=1.12-604)$. Though statistical significance was achieved, the small number of patients with a new postoperative deficit limits the reliability of the calculated odds ratio, i.e., twenty-six times increased risk.

\section{Discussion}

The purpose of this study was to review a large number of patients from a single surgeon's experience to assess the clinical utility of SSEP monitoring for cervical spine surgery. By evaluating the frequency with which SSEP data prompted an intraoperative intervention, this study sought to quantify how often the presence of SSEP monitoring actually made a difference in management and perhaps outcome. To our knowledge, the spinal cord monitoring data on our 807 cervical spine patients represents the largest series in the literature and is nearly double that of the next largest series. We believe that our large database provides cervical spine surgeons adequate information with which they can judge whether the time and expense of spinal cord monitoring is justified.

In our series of consecutive cervical spine operations, the use of SSEP monitoring led to an intervention in $2.1 \%$ of cases (17 of 809). In all cases, SSEP data deteriorated to a critical point, at which time, intraoperative maneuvers were instituted in an effort to improve the data. In most cases (14 of 17 patients, $82 \%$ ), the problem was adequately addressed through alteration of patient positioning, with tape and traction being the most common causes. When SSEP data improved back to baseline after such maneuvers, patients in this series (14 of 14 patients, 100\%) did well after surgery with no new neurological deficit. Conversely, our findings suggest that when alterations in patient positioning do not lead to an improvement back to baseline, the likelihood of neurologic sequelae is significantly greater. Thus, it is likely that in many cases, the intraoperative intervention prevented further neurologic injury.

Schwartz and colleagues published similar findings in a recent abstract. Reporting on 284 cases, a $6.0 \%(n=18)$ incidence of neurophysiologic data deterioration was identified during anterior cervical spine surgery. Repositioning and/or release of tape or traction resulted in rapid restitution of data? ${ }^{7}$ No comment was made regarding postoperative neurological status. Epstein and co-workers reported the usage of SSEP monitoring during 100 cervical operations. In their adequately monitored surgeries, they had no cases of quadriplegia or death, as compared with a $3.7 \%$ incidence of quadriplegia and a $0.5 \%$ mortality rate in the prior 218 patients who were unmonitored. Data from SSEP monitoring led to an intervention in 5 of 100 cases, all of which were due to hypotension; four of these patients were in a seated position ${ }^{1}$.

Another recent abstract reported on 12 out of 427 cases (2.8\%) that had an intraoperative loss of transcranial motorevoked potentials (TcMEP) and/or SSEP data ${ }^{10}$. Interventions included increasing mean arterial pressure to $90 \mathrm{mmHg}$, and high-dose steroids. One out of four patients with concomitant loss of TcMEP and SSEP data awoke with a dense paraparesis without significant recovery at three months. However, one out of eight patients with loss of TcMEP data with maintained SSEP data had transient postoperative weakness, which resolved within one week.

Previously published studies have established the value of SSEP monitoring of upper extremities during scoliosis surgery. O'Brien et al. ${ }^{4}$ prospectively monitored 151 patients for upper extremity SSEP changes, and found 78\% sensitivity for detecting postoperative sensory deficits, $100 \%$ sensitivity for combined sensory and motor deficits, and $98.5 \%$ specificity in predicting normal postoperative upper extremity function. Intraoperative traction in the prone position was associated with the three worst postoperative neurologic deficits, and all patients eventually recovered. Schwartz et al. ${ }^{5}$ showed 15 of 500 scoliosis patients (3\%) with impending brachial plexopathy, based on a $30 \%$ reduction in SSEP amplitude, though little data is provided regarding interventions and eventual outcome. Although surgeries for deformity correction tend to have much longer operative times and the patients are often qualitatively different, a similar cause and effect relationship between intraoperative monitoring, interventional maneuvers, and optimum neurological outcome is clearly present.

This study does have its limitations. The review of the data and outcomes is retrospective. However, the same sur- 
geon and the identical monitoring technique and warning criteria were utilized throughout the study period. Also, one might argue that an intraoperative deterioration of SSEP data may not necessarily represent a true neurological event. However, given that the data improved following an intervention, and that lack of improvement correlated with the presence of a new neurological deficit, it is reasonable to assume that the response to intraoperative SSEP information prevented neurological injury in the 15 of 17 presumed positive patients. Also, given that time is a critical factor in such intraoperative events, SSEP monitoring allowed expeditious intervention and improvement, whereas a wake-up test takes considerable time and carries its own risks, e.g., patient recall, self-extubation, and unpredictable patient movement during surgery.

Finally, it bears repeating that this study does not definitively demonstrate the need to perform SSEP monitoring in all cervical spine cases. It is notable that the actual incidence of neurological deficit is quite low, and that all deficits did resolve eventually. This study simply presents the results of a single institution, and the individual cervical spine surgeon may interpret that data and judge whether SSEP monitoring is a worthwhile surgical adjunct. Howev$\mathrm{er}$, as a direct result of our experience, we have incorporated these techniques as a standard of care in all routine and complex spine surgeries performed at our institution.

\section{REFERENCES}

1. Epstein N, Danto J, Nardi D: Evaluation of intraoperative somatosensory-evoked potential monitoring during 100 cervical operations. Spine 1993; 18: 737-747.

2. Bouchard JA, Bohlman HH, Biro C: Intraoperative improvements of somatosensory evoked potentials: correlation to clinical outcome in surgery for cervical spondylitic myelopathy. Spine 1996; 21: 589-594.

3. Bednarik J, Kadanka Z, Vohanka S, Stejskal L, Vlach
O, Schroder R: The value of somatosensory-and motorevoked potentials in predicting and monitoring the effect of therapy in spondylotic cervical myelopathy. Prospective randomized study. Spine 1999; 24: 1593-1598.

4. O'Brien MF, Lenke LG, Bridwell KH, Padberg A, Stokes M: Evoked potential monitoring of the upper extremities during thoracic and lumbar spinal deformity surgery: a prospective study. J Spinal Disord 1994; 7: 277284.

5. Schwartz DM, Drummond DS, Hahn M, Ecker ML, Dormans JP: Prevention of positional brachial plexopathy during surgical correction of scoliosis. J Spinal Disord 2000; 13: 178-182.

6. May DM, Jones SJ, Crockard HA: Somatosensory evoked potential monitoring in cervical surgery: identification of pre- and intraoperative risk factors associated with neurological deterioration. J Neurosurg 1996; 85: 566-573.

7. Schwartz D, Albert T, Hilibrand A, Vaccaro A: Neurophysiologic identification of impending neurologic injury from positioning for anterior cervical spine surgery. Presented at 29th Annual Meeting of the Cervical Spine Research Society, November 29, 2001, Monterey, California.

8. Gokaslan ZL, Samudrala S, Deletis V, Wildrick DM, Cooper PR: Intraoperative monitoring of spinal cord function using motor evoked potentials via transcutaneous epidural electrode during anterior cervical spinal surgery. J Spinal Disord 1997; 10: 299-303.

9. Darden BV 2nd, Hatley MK, Owen JH: Neurogenic motor evoked-potential monitoring in anterior cervical surgery. J Spinal Disord 1996; 9: 485-493.

10. Sethuraman V, Hilibrand A, Schwartz D, Vaccaro A, Albert A: The utility of transcranial motor-evoked potential monitoring as an indicator of impending cervical spinal cord injury. Presented at 29th Annual Meeting of the Cervical Spine Research Society, November 29, 2001, Monterey, California. 